\title{
Brownian motion and polarized three-dimensional quantum vacuum
}

\author{
D. Fiscaletti \\ Space Life Institute, San Lorenzo in Campo (PU), Italy. \\ e-mail: spacelife.institute@gmail.com
}

Received 17 November 2020; accepted 23 January 2021

\begin{abstract}
A nonlinear model of Brownian motion is developed in a three-dimensional quantum vacuum defined by a variable quantum vacuum energy density corresponding to processes of creation/annihilation of virtual particles. In this model, the polarization of the quantum vacuum determined by a perturbative fluctuation of the quantum vacuum energy density associated with a fluctuating viscosity, which mimics the action of dark matter, emerges as the fundamental entity which generates the Brownian motion.
\end{abstract}

Keywords: Brownian motion; three-dimensional quantum vacuum; variable quantum vacuum energy density; perturbative fluctuation of the quantum vacuum energy density.

PACS: 3.70.+k; 05.40.-a; 05.40.Jc; 11.; 12.60.-i.

DOI: https://doi.org/10.31349/RevMexFis.67.040706

\section{Introduction}

After the pioneering discovery of Robert Brown of the diffusive motion of granules of 1/4000th to 1/5000th of an inch in size, extracted from pollen grains of the plant Clarkia pulchella [1], a precise understanding of this phenomenon -since then known as Brownian motion- was established in the classic works of Albert Einstein [2], William Sutherland [3], Marian Smoluchowski [4], and Pierre Langevin [5]. On the basis of different arguments, they derived the linear time dependence of the mean squared displacement

$$
\left\langle r^{2}(t)\right\rangle=\int r^{2} P(\vec{r}, t) d V=2 d D t
$$

in $d$ spatial dimensions, and the Gaussian nature of the probability density function for a $\delta$-initial condition (Green's function)

$$
P(\vec{r}, t)=\frac{1}{(4 \pi D t)^{d / 2}} \exp \left(-\frac{r^{2}}{4 D t}\right) .
$$

In Eqs. (1) and (2), an important role is played by the diffusion coefficient $D$, which is given by the EinsteinSmoluchowski-Sutherland relation

$$
D=\frac{k_{B} T}{m \mu}=\frac{\left(R / N_{A}\right) T}{m \mu},
$$

where $m$ is the mass of the particle, $\eta$ is the viscosity of the ambient fluid, $R$ is the gas constant, $N_{A}$ is Avogadro's number. The diffusion coefficient (3) prompted a long series of relevant experiments on diffusive motion, such as Jean Perrin's systematic observations which provided the introduction of single particle tracking protocols [6,7], those due to Ivar Nordlund, who suggested time-resolved recordings using a moving film plate and thus getting around the need to average over ensembles of test particles [8], and those of Eugen Kappler, who first mapped out the Gaussian Boltzmann distribution of the equilibrium distribution of the angles to very high precision [9].
Recently, Cheng, Primulando and Spinrath introduced a novel approach to Brownian motion as an effect produced by the presence of dark matter. They showed that, by assuming that any movable target particle is actually in a bath of dark matter, if dark matter has some interaction with ordinary matter, the effect will be the generation of some random motion of the target particle, which is conceptually similar to Brownian motion [10]. On the other hand, recent research lead by the author of this paper suggests that dark matter does not exist as a primary physical reality but derives from opportune energy density fluctuations, associated to virtual pairs of particles-antiparticles, in a polarized three-dimensional (3D) quantum vacuum which is endowed with a fluctuating viscosity. In this way, in the light of the Cheng, Primulando and Spinrath insight, the suggestive perspective is opened that, at a fundamental level, Brownian motion emerges from the interaction between ordinary matter and a polarized 3D quantum vacuum characterized by a fluctuating viscosity. In this paper our aim is to explore this possibility.

The paper is structured as follows. In Sec. 2 we will review the foundations of the 3D quantum vacuum model developed by the author. In Sec. 3 we will develop the mathematical formalism which shows how the polarized 3D quantum vacuum endowed with a fluctuating viscosity induces Brownian motion on a target particle. In Sec. 4 we will apply the mathematical formalism of our 3D quantum vacuum model of Brownian motion in the context of a FriedmannRobertson-Walker (FRW) spatially flat geometry. In Sec. 5, we will summarize the results of the paper.

\section{The foundations of the three-dimensional quantum vacuum model with a fluctuating viscosity}

In order to see why and in what sense the Brownian motion of a particle can be considered as an emerging phenomenon 
from a polarized 3D quantum vacuum characterized by a fluctuating viscosity, we need first to realize the interpretation of ordinary particles of the Standard Model and the concept of polarized 3D quantum vacuum characterized by a fluctuating viscosity in the light of some current research.

The model of 3D quantum vacuum proposed by the author in a series of recent works [11-20], postulates that the appearance of ordinary matter derives from an opportune excited state of the 3D quantum vacuum defined by the quantum vacuum energy density

$$
\rho=\rho_{p E}-\frac{m c^{2}}{V}
$$

where the Planck energy density

$$
\rho_{p E}=\frac{m_{p} C^{2}}{l_{p}^{3}}=4,641266 \times 10^{113} \mathrm{~J} / \mathrm{m}^{3},
$$

can be defined as the ground state of the 3D quantum vacuum, and $m$ and $V$ are the mass and volume of the particle, respectively.

The excited state of the 3D quantum vacuum corresponding to the appearance of this material particle is therefore characterized by the change of the energy density

$$
\Delta \rho_{q v E} \equiv \rho_{p E}-\rho=\frac{m c^{2}}{V},
$$

with respect to the ground state. Moreover, the model predicts that ordinary matter emerges from elementary reduction-state (RS) processes of creation/annihilation of virtual particles of the vacuum medium. In particular, in epistemological affinity with a recent approach proposed by Licata and Chiatti where quantum jumps are processes of entry and exit from the usual temporal domain to a timeless vacuum [21,22], a real quantum massive particle of the Standard Model is here given by the sum of a bare mass produced by the virtual particles of the 3D quantum vacuum, and an additional term associated with the self-interaction, which is responsible of the actual appearance, visibility, tangibility of the particle.

This model of the 3D quantum vacuum, despite the consideration of a series of specific hypotheses regarding the relations between the states of the vacuum and a variable quantum vacuum energy density, has the merit to suggest interesting perspectives of unification of gravity and quantum and of a completion of the Standard Model where the action of the Higgs boson emerges as the interplay of opportune fluctuations of the quantum vacuum energy density (see, in particular, [20]).

On the other hand, on the basis of the results obtained in [23], in our model of 3D quantum vacuum, the action of dark matter, which is invoked to explain the rotation curves of galaxies, is assimilated to a more fundamental concept of polarization of the vacuum characterized by a fluctuating viscosity. In this regard, in our theory, the crucial point is that the flat rotation curves of the spiral galaxies may be explained in terms of fundamental fluctuations of the quantum vacuum energy density (and therefore of peculiar excited states of the $3 \mathrm{D}$ vacuum), in the range of the ultra-low frequencies, corresponding to excited states of the $3 \mathrm{D}$ quantum vacuum which do not act as a perfect superfluid medium, but are characterized by a certain (fluctuating) viscosity of the form

$$
\mu(t)=\mu \cos (\Omega t),
$$

and thus correspond to RS processes which produce only the bare mass of the virtual particles (namely without the selfinteraction which is able to make the particles visible).

The polarization of the 3D quantum vacuum is due to $R S$ processes involving a background of elementary perturbative mass $\mu \hbar / n l_{p}^{2} \Delta \rho_{q v E_{0}}$ (where $n$ is the number of the virtual particles in the volume into consideration and $\Delta \rho_{q v E_{0}}$ is the change of the quantum vacuum energy generating the appearance of matter at a rest mass in its bare state) determined by a perturbative fluctuation of the quantum vacuum energy density given by relation [23]

$$
\Delta \rho_{\text {perturbative }}=\frac{\mu \hbar c^{2}}{n V l_{p}^{2} \Delta \rho_{q v E_{0}}} .
$$

As regards the explanation of the flat rotation curves of the spiral galaxies, the perturbative fluctuation of the quantum vacuum energy density (8) can mimic the action of dark matter in the sense that it provokes an exchange of the energy of the rotating galactic matter with the quantum vacuum fluctuations, which generates, in regime ultra-low frequencies, a sort of breathing of the galaxy which can be described by the small fluctuations in time of the orbital speed

$$
V(r, t)=\frac{\Gamma}{2 r n} \sum_{i=1}^{n}\left(1-\exp \left[-\frac{r^{2}}{\sum_{n}(t)}\right]\right),
$$

where

$$
\sum_{n}(t)=4\left(\frac{\Delta \rho_{\text {perturbative }} V l_{p}^{2}}{\hbar \Omega_{n}^{2}}\right) \sin \left(\Omega_{n} t\right)+\sigma_{n}^{2} .
$$

In Eqs. (9) and (10), the parameter $\Omega_{n}$ is an oscillation frequency while the parameter $\sigma_{n}$ is given by $\sigma_{n}=4 c / \Omega_{m}$. These two parameters determine a wide spectrum of the viscosity coefficients which is discrete with equidistant position of each component and is condensed in the point $\Omega=0$ and imply that the strongest contribution to the vorticity gives modes with frequencies close to zero. On the basis of the treatment provided in [23], under the constraints $\Gamma=10^{27} \mathrm{~m}^{2} / \mathrm{s}, \Omega_{n}=10^{-11} \mathrm{~s}^{-1}$, and $n=25$, the parameter $\sigma_{n}=4 c / \Omega_{n}$ ranges from 10000 to 300000 light years, which reproduces the diameter of the ordinary spiral galaxies and the orbital speed (9) determined by the perturbative fluctuation of the quantum vacuum energy density (8) can explain the stabilized behaviour of the speed of the arms of spiral galaxies, with increasing distance from the core of the galaxy, compatibly with the experimental observations (and in agreement with the results obtained by Sbitnev in [24]), 
reproducing the observed flattening of the orbital speeds of spiral galaxies, in terms just of the fluctuations of virtual particles of the vacuum, namely of the polarization of the vacuum generated by its fluctuating viscosity.

It must be emphasized that the introduction of a polarized quantum vacuum characterized by a fluctuating viscosity as the ultimate origin of the behaviour of the rotation curves of the spiral galaxies can be considered physically relevant (and somewhat necessary, according to our point of view) for at least two orders of motivations. On one hand, because of the inability of dark matter to explain the so-called Baryonic Tully-Fisher Relationship

$$
M_{B}=A v^{4},
$$

where

$$
A=(47 \pm 6) \quad \mathrm{M}_{\odot} \mathrm{km}^{-4} \mathrm{~s}^{4},
$$

between the baryonic mass of the galaxy $M_{B}$ and the galaxy's constant outer rotational velocity $v$. On the other hand, because Milgrom's Modified Newtonian Dynamics [25-27], the most prominent alternative approach to dark matter, that postulates the inertia of an object varies with acceleration in a manner that reproduces the Baryonic Tully-Fisher Relationship and turns out to be in good agreement with the galactic rotational curves, however is not able to explain in a natural way the flatness of our universe, still requiring the existence of dark matter in order to explain the accelerated expansion of space. Instead, our approach of polarized quantum vacuum characterized by a perturbative fluctuation of the quantum vacuum energy density can face these issues in a natural way. Moreover, it is compatible with the results of other interesting approaches existing in the current literature which provide an alternative to dark matter and Modified Newtonian Dynamics. For example, in a series of papers [2832], Penner proposed the idea that the gravitational field of a baryonic mass induces an energy contribution from the vacuum which leads naturally to the Baryonic Tully-Fisher Relationship [28], leads to excellent agreement with the rotational curves of galaxies [29, 30] as well as the velocity dispersion and shear measurements taken with the Coma cluster [31]. In an analogous spirit, in [33] Roshan and Rahvar studied the dynamics of large-scale structures, such as the time evolution of exponential disk galaxies, in the context of a non-local theory of gravity. Our theory of 3D quantum vacuum characterized by a perturbative fluctuation of the quantum vacuum energy density has epistemological affinities with the background of these models of explanation of dynamics of galaxies which are alternative to dark matter and therefore can be considered as a physically plausible starting-point in order to throw new light as regards the description of the dynamics of large-scale structures and to build novel scenarios in our cosmological theories.

\section{Brownian motion induced by the polarized three-dimensional quantum vacuum with a fluctuating viscosity}

After seeing what is ordinary matter and what is the consequence of the polarization of the vacuum in presence of a fluctuating viscosity, we can now analyse how emerges the Brownian motion of a material particle in this background. When a test particle moves in space, it has the possibility to meet regions of space characterized by a fluctuating viscosity and thus to interact with regions of the 3D quantum vacuum which are in the specific peculiar excited state associated with the perturbative fluctuation of the 3D quantum vacuum (8). In order to describe the interaction between the dressed massive test particle and the perturbative fluctuation of the quantum vacuum (8), one can introduce the diffusion coefficient:

$$
D=\frac{\hbar^{2} c^{2} n}{2 \Delta \rho_{\text {perturbative }} V} .
$$

Taking account of (8), Eq. (11) can be expressed as

$$
D=\frac{\hbar n^{2} l_{p}^{2} \Delta \rho_{q v E_{0}}}{2 \mu} .
$$

In this way, the probability density function of Gaussian nature, invoked by the original treatments of Albert Einstein [2], William Sutherland [3], Marian Smoluchowski [4] and Pierre Langevin [5], in our approach reads

$$
\begin{aligned}
P(\vec{r}, t) & =\frac{1}{\left(4 \pi \frac{\hbar^{2} n^{2} l_{p}^{2} \Delta \rho_{q v E_{0}}}{2 \mu \hbar}\right)^{d / 2}} \\
& \times \exp \left(-\frac{r^{2}}{4 \frac{\hbar n^{2} l_{p}^{2} \Delta \rho_{q v E_{0}}}{2 \mu} t}\right),
\end{aligned}
$$

and the Einstein-Smoluchowski-Sutherland relation becomes

$$
\frac{\hbar n^{2} l_{p}^{2} \Delta \rho_{q v E_{0}}}{2 \mu}=\frac{k_{B} T}{m \eta}=\frac{\left(R / N_{A}\right) T}{m \eta},
$$

which yields

$$
\eta=\frac{2 \mu k_{B} T}{m \hbar n^{2} l_{p}^{2} \Delta \rho_{q v E_{0}}} .
$$

In other words, in our theory of the Brownian motion, the real macroscopic viscosity of the ambient fluid $\eta$ can be seen as a collective property which is generated by more fundamental properties of the 3D quantum vacuum, i.e. the perturbative fluctuation of the quantum vacuum energy density which describes the polarization of the vacuum determined by a fluctuating viscosity.

Now, the crucial point regarding how the Brownian motion of a test particle emerges in the polarized vacuum with a fluctuating viscosity, invoked by our theory, is the following. The ultimate source of the Brownian motion is the perturbative fluctuation of the quantum vacuum energy density (8), 
which can be seen as an external potential acting on the test particle, as a sort of polarized bath inside which the test particle moves and which therefore produces a friction onto the particle.

Now, in order to provide a mathematical treatment of the Brownian motion inside our model, we will make our calculations, for sake of simplicity, inside a geodesic picture for a test particle instead of using the hydrodynamic flows of a typical fluid volume element. This simplification may be considered physically plausible by virtue of the fact that the geodesics for a test particle in the polarized 3D quantum vacuum are dynamically equivalent to the hydrodynamic flows in the interior of a fluid volume element of the same vacuum. In fact, in the model of the 3D quantum vacuum, as shown by the author of this paper in [14], the perturbative fluctuation of the quantum vacuum energy density (12) mimicking the action of dark matter in the explanation of the observed flattening of the orbital speeds of the spiral galaxies in a geodesic picture, may be associated to a energy-momentum tensor of the form

$$
T^{\mu \nu}=(\varepsilon+p) u^{\mu} u^{\nu}+p \eta^{\mu \nu}+\Pi^{\mu \nu},
$$

where $\varepsilon$ and $p$ are functions per unit volume expressed in units of pressure and $\eta^{\mu \nu}$ is the metric tensor having the spacelike signature $(-,+,+,+)$. In Eq. (16) the crucial term is $\Pi^{\mu \nu}$ which describes the viscosity of the vacuum in a hydrodynamic picture. In other words, we can say that the term $\Pi^{\mu \nu}$ indicates that the 3D quantum vacuum acts, in general, as a non-perfect fluid medium characterized by a given degree of viscosity, and can be considered as the hydrodynamic counterpart of the perturbative fluctuation of the quantum vacuum energy density (8). By virtue of the correspondence between the term $\Pi^{\mu \nu}$ and the perturbative fluctuation of the quantum vacuum energy density, the motion of a test particle in the 3D quantum vacuum treated in terms of geodesics which emerge from the interaction with the perturbative fluctuation of the quantum vacuum energy density, turns out to be dynamically equivalent to its motion in terms of hydrodynamic flows resulting from the interaction with the viscosity term $\Pi^{\mu \nu}$ of a typical fluid element of the vacuum. In particular, the orbital speed (9) determined by the perturbative fluctuation of the quantum vacuum energy density may be seen as a collective hydrodynamic property which emerges from the fundamental energy-momentum tensor (16) in the sense that it corresponds to a vorticity $\vec{\omega}=\nabla \times \vec{V}$ of the vacuum satisfying equation

$$
\frac{\partial \vec{\omega}}{\partial t}+(\vec{\omega} \cdot \nabla) \vec{V}=\frac{\mu(t) c^{2}}{\Delta \rho_{q v E_{0}}} \nabla^{2} \vec{\omega}
$$

which derives directly from the following generalized Navier-Stokes equation for the excited states of the 3D quan- tum vacuum

$$
\begin{aligned}
\frac{V(\varepsilon+P)}{n} \gamma & \left(\frac{1}{c} \frac{\partial v}{\partial t}+[\vec{v} \cdot \nabla \vec{v}]\right)+\nabla Q-\frac{v}{c} \frac{\partial Q}{\partial t} \\
& +\frac{\partial(V \mu(t) / n)}{\partial t} \cdot\left(\pi^{0, i}-v \pi^{0,0}\right) \\
& \left.+\frac{V \mu(t)}{n}\left(\partial_{(} \mu\right) \pi^{\mu, i}-\vec{v} \partial_{\mu} \pi^{\mu, 0}\right) \\
& +\frac{V \mu(t)}{n}\left(\partial_{\mu} \pi^{\mu, i}-\vec{v} \partial_{\mu} \pi^{\mu, 0}\right)=0 .
\end{aligned}
$$

A for Eq. (17) and (18), $\vec{V}=c \vec{v}$ is the real velocity of the fluid associated with the fluctuations of the quantum vacuum, while $Q$ is the quantum potential which describes the influence through the pressures that arise between ensembles of virtual particles populating the vacuum and is given by relation

$$
\begin{aligned}
Q & =V \frac{p_{1}+p_{2}}{n}=-\frac{D^{2}}{c^{2}}\left(\nabla^{2} \Delta \rho_{q v E}-\frac{1}{c^{2}} \frac{\partial^{2}}{\partial t^{2}}\right) \\
& +\frac{D^{2}}{2 \Delta \rho_{q v E_{0}} c^{2}}\left(\left[\nabla \Delta \rho_{q v E_{0}}\right]^{2}-\frac{1}{c^{2}}\left[\frac{\partial}{\partial t} \Delta \rho_{q v E_{0}}\right]^{2}\right) \\
& =-\frac{\hbar^{2} c^{2} n}{2 \Delta \rho_{q v E_{0}} V} \frac{\partial_{\mu} \partial^{\mu} R}{R},
\end{aligned}
$$

where $R$ is the square root of the density distribution of the virtual particles in the vacuum and $D$ is the diffusion coefficient given by

$$
D=\frac{\hbar c^{2} n}{2 \Delta \rho_{q v E_{0}} V} .
$$

In light of the considerations we have made here, there is a direct physical correspondence between the geodesics emerging from the interaction with the perturbative fluctuation of the quantum vacuum energy density and the hydrodynamic flows in the vacuum acting as a non-perfect fluid. As a consequence, we can develop our treatment of the Brownian motion of a test particle in the 3D polarized quantum vacuum, as a toy-model inside a geodesic picture, without the necessity to use a hydrodynamic picture.

Now, our toy-model of the Brownian motion of a test particle in the 3D polarized quantum vacuum can be mathematically formulated by starting from the following Schrödinger equation for the coupled system test particle+perturbative fluctuation of the quantum vacuum energy density:

$$
i \hbar \frac{\partial \psi}{\partial t}=\left(\frac{\hat{p}^{2}}{2 m}+U+\frac{\mu \hbar c^{2}}{n l_{p}^{2} \Delta \rho_{q v E_{0}}}+H_{\Delta \rho_{q v E_{0}}}\right) \psi
$$

where $\psi$ is the wave function of the system, $m$ is the mass of the Brownian particle, $\hat{p}$ is the momentum operator of the Brownian particle, $U(r)$ is an external potential acting on the Brownian particle only (due to the interaction with the virtual sub-particles of the 3D quantum vacuum), $\left(\mu \hbar c^{2} / n l_{p}^{2} \Delta \rho_{q v E_{0}}\right)$ is the perturbative potential due 
to the fluctuating viscosity of the vacuum accounting for the particle-bath interaction and $H_{\Delta \rho_{q v E_{0}}}$ is a pure bath Hamiltonian (associated with the polarized vacuum). By virtue of its statistical nature, the wave function which is solution of (21) can be expressed as $\psi=\Phi \phi$, where $\phi$ is the wave function of the Brownian particle and $\Phi$ is the conditional wave function of the perturbative fluctuation of the quantum vacuum energy density. By following the treatment of Tsekov in [34], if one substitutes $\psi=\Phi \phi$ in Eq. (21), after some mathematical manipulations, one obtains:

$$
\begin{aligned}
i \hbar \frac{\partial \phi}{\partial t} & =\left(\frac{\bar{p}^{2}}{2 m}+U+\frac{\mu \hbar c^{2}}{n l_{p}^{2} \Delta \rho_{q v E_{0}}}\right. \\
& \left.-T S+\frac{b A}{m}-\frac{b(\hat{p} \cdot r+r \cdot \hat{p})}{2 m}\right) \phi .
\end{aligned}
$$

Equation (22) turns out to be a nonlinear Schrödinger equation where $T$ is the temperature, $S$ is the Boltzmann entropy of the particle, $b$ is the friction coefficient of the particle associated with its interaction with the polarized vacuum (which measures the resistance produced by the polarized vacuum to the Brownian motion of the test particle), $A=\int p \cdot d r$ is the action (which depends on the particle wave function $\phi$ ). The quantity $\left(\mu \hbar c^{2} / n l_{p}^{2} \Delta \rho_{q v E_{0}}\right)-T S$ means that the work required to remove quasi-statistically the quantum Brownian particle from the bath of the polarized vacuum at constant temperature and volume depends on the perturbative fluctuation of the quantum vacuum energy density. In the light of Eq. (22), the polarized bath determined by the perturbative fluctuation of the quantum vacuum energy density (12) not only decreases the kinetic energy of the test particle but modifies its energy spectrum too because of the term $b A / m$. Moreover, as regards the friction coefficient $b$, observations of rotation curves of spiral galaxies can set the bounds. In fact, in order to reproduce the observed flattening of the rotation curves of spiral galaxies, a reasonable estimate for the friction coefficient appearing in Eq. (22) is represented by the value $b \sim m \Omega_{n}$ and, thus, if one assumes the value $10^{-6} \mathrm{Kg}$ for the mass of the test material particle, one has $b \sim 10^{-17} \mathrm{Kg} \cdot \mathrm{s}^{-1}$.

In order to explore the consequences of the non-linear Schrödinger Eq. (22) - and in order to study its limit cases $T \rightarrow 0$ and $T \rightarrow \infty$ - we recast this equation by the Madelung transformation, writing the wave function as

$$
\psi=R e^{i A / \hbar} \text {. }
$$

By substituting (23) into Eq. (22) and separating real and imaginary parts, one obtains a continuity equation

$$
\frac{\partial \rho}{\partial t}+\frac{\hbar}{m} \nabla(\rho \nabla A)=0,
$$

and the Hamilton-Jacobi equation

$$
\begin{aligned}
m \frac{\partial}{\partial t} V & +m V \cdot \nabla V+b V= \\
& -\nabla\left(Q+U+\frac{\mu \hbar c^{2}}{n l_{p}^{2} \Delta \rho_{q v E_{0}}}-T S\right),
\end{aligned}
$$

where $V=(\nabla A-b r) / m, \rho=n / \Delta V$, where $n$ is the number of the virtual particles in the volume $\Delta V$ and

$$
Q=\frac{P_{1}+P_{2}}{\rho}=\frac{\hbar^{2}}{8 m}\left(\frac{\nabla \rho}{\rho}\right)^{2}-\frac{\hbar^{2}}{4 m} \frac{\nabla^{2} \rho}{\rho}
$$

is the quantum potential which is responsible of quantum effects and thus of non-local correlations in this background. In the light of the results obtained in [20], the quantum potential (26) can also be expressed in a similar form to (19), namely as

$$
\begin{aligned}
Q & =V \frac{p_{1}+p_{2}}{\rho}=-\frac{\hbar^{2} c^{2} n^{2}}{4 \Delta \rho_{q v E_{0}}^{2} V^{2}} \\
& \times\left(\nabla^{2} \Delta \rho_{q v E_{0}}-\frac{1}{c^{2}} \frac{\partial^{2}}{\partial t^{2}} \Delta \rho_{q v E_{0}}\right)+\frac{\hbar^{2} c^{2} n^{2}}{8 \Delta \rho_{q v E_{0}}^{3} V^{2}} \\
& \times\left(\left[\nabla \Delta \rho_{q v E_{0}}\right]^{2}-\frac{1}{c^{2}}\left[\frac{\partial}{\partial t} \Delta \rho_{q v E_{0}}\right]^{2}\right)
\end{aligned}
$$

Equation (27) shows that the quantum potential of the vacuum describes the geometry via the pressures $p_{1}$ and $p_{2}$ that arise by the collisions between the virtual particles populating the vacuum. In this picture, the collective excitations of the virtual particles of the physical vacuum (which provide the polarized bath determining the Brownian motion of the test ordinary particle) define therefore a mesoscopic level which is characterized by a degree of non-locality. The delocalization degree regarding the mesoscopic level of these collective excitations may also be evaluated by defining a Bell length of the vacuum ultimately associated with the osmotic pressure arising as a consequence of the motion of the virtual particles in the vacuum:

$$
L_{\text {vacuum }}=\frac{2}{\sqrt{-\left(\frac{\nabla \rho}{\rho}\right)^{2}+2 \frac{\nabla^{2} \rho}{\rho}}},
$$

which, by following [20], may be expressed as

$$
L_{\text {quantum }}=\frac{c^{2} \hbar}{D n \sqrt{\frac{V}{n}\left(-\left[\nabla \Delta \rho_{q v E_{0}}\right]^{2}+\frac{1}{c^{2}}\left[\frac{\partial}{\partial t} \Delta \rho_{q v E_{0}}\right]^{2}-\Delta \rho_{q v E_{0}}\left[\nabla^{2} \Delta \rho_{q v E_{0}}-\frac{1}{c^{2}} \frac{\partial^{2}}{\partial t^{2}} \Delta \rho_{q v E_{0}}\right]\right)}}
$$


where $D_{n}=\left(c^{2} n \hbar\right) / 2 \Delta \rho_{q v E_{0}} V$.

On the basis of relation (29), the points where the quantum potential (27) tends to zero -which correspond to an infinite value of the Bell length of the vacuum (29)- indicate the boundary of the region where the virtual particles of the vacuum are delocalized. Therefore the following re-reading of the motion of the test particle in the bath provided by the polarized 3D vacuum becomes permissible: the virtual particles of the vacuum which are associated with a perturbative fluctuation determined by a fluctuating viscosity generate a Brownian motion of the test particle and the interaction between the test particle and the virtual particles of the medium implies that the background of the processes can be described by a quantum potential of the vacuum (27) and thus by a Bell length of the vacuum (29), which turns out to be exclusively dependent on the rapidity of the variation of the quantum potential (27) in the entire set of coordinates of the configuration space and can be considered as a measure of the spatial length on which non-local effects of the vacuum are generated in a semi-classical description.
In light of Eqs. (27) and (29) one can evaluate the conditions in which the contributions of the quantum potential, describing the quantum effects in the Brownian motion -which are negligible for all practical purposes in the thermodynamic limit- become relevant. In this regard, one can use the consideration that, in the light of some recent research, the maximum de-localization of a quantum system corresponds to the value 1 of the Bell length [22]. By applying this constraint, one obtains the following simple relation which are satisfied by the number of virtual particles-antiparticles of the RS processes of the 3D quantum vacuum in the condition of maximum entanglement, and thus when the quantum effects described by the quantum potential become relevant:

$$
2 \frac{\nabla^{2} \rho}{\rho}-\left(\frac{\nabla \rho}{\rho}\right)^{2}=4,
$$

which, by taking account of relation (29), may be written as

$$
n=\frac{16 \Delta \rho_{q v E_{0}}^{2} V}{\left(-\left[\nabla \Delta \rho_{q v E_{0}}\right]^{2}+\frac{1}{c^{2}}\left[\frac{\partial}{\partial t} \Delta \rho_{q v E_{0}}\right]^{2}-\Delta \rho_{q v E_{0}}\left[\nabla^{2} \Delta \rho_{q v E_{0}}-\frac{1}{c^{2}} \frac{\partial^{2}}{\partial t^{2}} \Delta \rho_{q v E_{0}}\right]\right)}
$$

Now, in the limit of zero temperature, the entropic term in Eq. (22) drops out and the solutions of Eqs. (24) and (25) are

$$
\rho=\frac{\exp \left(-\frac{(x-\xi)^{2}}{2 \sigma_{x}^{2}}\right)}{\sqrt{2 \pi \sigma_{x}^{2}}}
$$

and

$$
V=\frac{\partial \xi}{\partial t}+(x-\xi) \frac{1}{\sigma_{x}} \frac{\partial \sigma_{x}}{\partial t}
$$

where the mean value $\xi$ and dispersion $\sigma_{x}$ obey the following dynamic equations

$$
\begin{aligned}
& m \frac{\partial^{2}}{\partial t^{2}} \xi+b \frac{\partial}{\partial t} \xi=\frac{-U-\frac{\mu \hbar c^{2}}{n l_{p}^{2} \Delta \rho_{q v E_{0}}}}{x}, \\
& m \frac{\partial^{2}}{\partial t^{2}} \sigma_{x}^{2}+b \frac{\partial}{\partial t} \sigma_{x}^{2}=\frac{\frac{\hbar^{2}}{4 m \sigma_{x}^{3}}}{x} .
\end{aligned}
$$

Equation (34) can be considered as a sort of generalized version of the Ehrenfest theorem in the polarized vacuum. Equation (35) is a nonlinear equation by virtue of the presence of the quantum potential. In the simple case of absence of friction effects of the polarized vacuum (namely $b=0$ ) the solution of Eqs. (34) and (35) is given by $\sigma_{x}^{2}=$ $\sigma_{x}^{2}(0)+\left(\hbar t / 2 m \sigma_{x}^{2}(0)\right)^{2}$ which describes the spreading of a Gaussian wave packet. Instead, in the case of strong friction produced by the polarized vacuum, the first inertial term in Eqs. (34) and (35) is negligible with respect to the other terms and the solution is $\sigma_{x}^{2}=\hbar \sqrt{t / m b}$; in the limit $b \rightarrow \infty$ the initial state will last forever because the Brownian particle is constrained in a kinetic well and, as a consequence of the Heisenberg relations, in the quantum regime one has a restriction of the relaxation of the momentum dispersion.

However, in general, the temperature, which is introduced in Eq. (22) as a parameter describing the chaotic motion of the bath virtual particles of the polarized vacuum, is the local non-equilibrium temperature. When the temperature is increasing, one expects an increasing of the chaotic motion of the virtual particles of the polarized vacuum. In the limit of $T \rightarrow \infty$, the energetic scenario of non-equilibrium thermodynamics corresponds to a throughput of heat, namely a kinetic energy at the subquantum level, and one expects that the test Brownian particle will not only receive kinetic energy from the thermodynamic environment, but, in order to balance the stochastic influence of the supplementary momentum fluctuations determined by the interaction with the perturbative fluctuation of the quantum vacuum energy density, it will also dissipate heat into the environment. On the basis of the "vacuum fluctuation theorem" introduced in [35], the larger the energy fluctuation of the oscillating "system of interest" is, the higher is the probability that heat will be dissipated into the environment rather than be absorbed. 
As a consequence of the motion of a very fast Brownian particle, one has a complete destruction of the local thermodynamic equilibrium. Therefore, we must focus the attention on the relatively low velocities in order to be able to employ the equilibrium statistical temperature, which occurs when the interaction of the test particle with the perturbative fluctuation of the quantum vacuum energy density determines a linear response. Under these constraints, one can linearize Eq. (22) on $V$ obtaining

$$
\begin{aligned}
m \frac{\partial^{2}}{\partial t^{2}} \rho & =\nabla \cdot\left(b \rho V+\rho \nabla\left[Q+U+\frac{\mu \hbar c^{2}}{n l_{p}^{2} \Delta \rho_{q v E_{0}}}-T S\right]\right. \\
& +m \nabla \cdot[\rho V V])=-b \frac{\partial p}{\partial t}+\nabla \cdot(\rho \nabla[Q+U \\
& \left.\left.+\frac{\mu \hbar c^{2}}{n l_{p}^{2} \Delta \rho_{q v E_{0}}}-T S\right]\right)+O\left(V^{2}\right) .
\end{aligned}
$$

On the basis of the treatment of Tsekov in [34], since the thermodynamic entropy

$$
S_{t}=-\frac{\partial}{\partial T}\left(Q+U+\frac{\mu \hbar c^{2}}{n l_{p}^{2} \Delta \rho_{q v E_{0}}}-T S\right)_{p . b}
$$

is the temperature derivative of the free energy, one can express

$$
\left(Q+U+\frac{\mu \hbar c^{2}}{n l_{p}^{2} \Delta \rho_{q v E_{0}}}-T S\right)
$$

by integration on $T$, thus obtaining:

$$
\begin{aligned}
m \frac{\partial^{2}}{\partial t^{2}} \rho & +b \frac{\partial \rho}{\partial t}=K_{B} T \nabla \\
& \times\left(\rho \nabla \int_{0}^{\beta} \frac{1}{\sqrt{\rho}}\left[\hat{H}+2 \frac{\partial}{\partial \beta}\right] \sqrt{\rho} d \beta\right)_{b},
\end{aligned}
$$

where the subscript $b$ indicates that in this relation the friction coefficient $b$ is considered constant during the integration on $\beta$. Equation (37) is a non-linear differential equation describing the quantum Brownian motion beyond the linear response. Instead, if the friction coefficient is not constant, and for example depends on the temperature, this means that the interaction of the Brownian particle with the polarized vacuum corresponds to variable fluctuations of the quantum vacuum energy density which generate the maximum degree of chaotic motion of the Brownian particle (and, in this regard, further research is obviously required in order to clarify the physical details of these processes).

Moreover, at zero temperature, Eq. (37) becomes the following non-linear equation describing a purely quantum diffusion

$$
\begin{aligned}
m \frac{\partial^{2}}{\partial t^{2}} \rho & +b \frac{\partial \rho}{\partial t}=\nabla \\
& \times\left(\rho \nabla\left[Q+U+\frac{\mu \hbar c^{2}}{n l_{p}^{2} \Delta \rho_{q v E_{0}}}\right]\right) .
\end{aligned}
$$

According to Eq. (39) the quantum diffusion associated with the Brownian motion of the test particle in the polarized bath is guided by the quantum potential and is influenced by the mediatory action of the perturbative fluctuation of the quantum vacuum energy density. In the case of strong friction, Eq. (38) implies a dispersion-dependent quantum diffusion coefficient of the form

$$
D_{Q}=\frac{\hbar^{2}}{4 m b \sigma_{x}^{2}} .
$$

By substituting (12) into (39), one finds

$$
\sigma_{x}^{2}=\frac{\hbar \mu}{2 m n^{2} l_{p}^{2} \Delta \rho_{q v E_{0}}},
$$

namely the dispersion of the wave packet turns out to be a collective property generated by the polarized vacuum characterized by a fluctuating viscosity, i.e. by the perturbative fluctuation of the quantum vacuum energy density.

As regards the problem of finding the exact solutions of Eq. (37), there are obvious mathematical difficulties. However, a reasonable assumption is that, at the first order, the external potential is a harmonic one of the form $U=m \omega_{0}^{2} x^{2} / 2$ where

$$
\omega_{0}=\frac{2 \Delta \rho_{q v E_{0}}}{\hbar n},
$$

is the frequency associated with the motion of the virtual subparticles of the vacuum, namely

$$
U=\frac{2 \Delta \rho_{q v E_{0}}^{2} V^{2} m x^{2}}{\hbar^{2} n^{2}},
$$

and that the polarized bath represented by the perturbative fluctuation of the quantum vacuum energy density provides a correction in terms of dissipative, dispersive effects. Under these constraints, the general solution of the non-equilibrium Eq. (37) will be given by a Gaussian distribution at the lowest order, plus corrections expressed by dissipative, dispersive terms linked with the perturbative fluctuation of the quantum vacuum energy density:

$$
\begin{gathered}
\rho=\frac{\exp \left(-[x-\xi]^{2} / 2 \sigma_{x}^{2}\right)}{\sqrt{2 \pi \sigma_{x}^{2}}}-\frac{(x-\xi)}{\sqrt{2 \frac{\hbar n^{2} l_{p}^{2} \Delta \rho_{q v E_{0}}}{2 \mu} t}} \\
-\frac{(x-\xi)^{2}}{\sqrt{4 \frac{\hbar n^{2} l_{p}^{2} \Delta \rho_{q v E_{0}}}{2 \mu}} t}-\frac{(x-\xi)^{3}}{12 \sqrt{2\left(\frac{\hbar n^{2} l_{p}^{2} \Delta \rho_{q v E_{0}}}{2 \mu} t\right)^{3}}-\ldots}
\end{gathered}
$$

and

$$
V=\frac{\frac{\partial}{\partial t} \xi+(x-\xi) \frac{\partial}{\partial t} \sigma_{x}}{\sigma_{x}}
$$


The corresponding mean value $\xi$ and dispersion $\sigma_{x}$ satisfy the following dynamic equations

$$
\begin{aligned}
& m \frac{\partial^{2}}{\partial t^{2}} \xi+b \frac{\partial}{\partial t} \xi+m \omega_{0}^{2} \xi=\frac{\mu \hbar c^{2}}{n l_{p}^{2} \Delta \rho_{q v E_{0}}} \\
& m \frac{\partial^{2}}{\partial t^{2}} \sigma_{x}^{2}+b \frac{\partial}{\partial t} \sigma_{x}^{2}+2 m\left(\omega_{0}^{2}-k_{B} T\right. \\
&\left.\times \int_{0}^{\beta} \frac{\hbar^{2}}{4 m^{2} \sigma_{x}^{4}} d \beta\right)_{b} \sigma_{x}^{2}=2 k_{B} T .
\end{aligned}
$$

Despite the mathematical difficulties, Eqs. (45) and (46) lead to obtain the equilibrium dispersion

$$
\sigma_{x}^{2}=\left(\frac{\hbar}{2 m \omega_{0}}\right) \operatorname{coth}\left(\frac{\beta \hbar \omega_{0}}{2}\right),
$$

which coincides with the results of statistical thermodynamics, where now $\omega_{0}$ is given by Eq. (41), namely

$$
\sigma_{x}^{2}=\left(\frac{\hbar^{2} n}{4 m \Delta \rho_{q v E_{0}}}\right) \operatorname{coth}\left(\frac{\beta \Delta \rho_{q v E_{0}}}{n}\right) .
$$

In other words we can say that, in our model, the equilibrium dispersion emerges as a collective property which is generated by the fundamental frequencies of the 3D quantum vacuum, given by (41).

Moreover, if one considers the case of a free Brownian particle $\left(\omega_{0}=0\right)$ in the high friction limit relevant to the Einstein law of Brownian motion, Eqs. (40) and (41) become the following one:

$$
\frac{\partial \sigma_{x}^{2}}{\partial t}=2 D\left(1+\sigma_{x}^{2} \int_{0}^{\beta} \frac{\hbar^{2}}{4 m \sigma_{x}^{4}} d \beta\right)_{b}
$$

where $D=\left(k_{B} T\right) / b=\left(\hbar n^{2} l_{p}^{2} \Delta \rho_{q v E_{0}}\right) / 2 \mu$ is the Einstein diffusion coefficient, which here can be seen as a collective property emerging from a more fundamental perturbative fluctuation of the quantum vacuum energy density describing the polarized vacuum. In the light of Eq. (48), we can say that the quantum potential, and thus the pressure provoked by the virtual particles of the vacuum, determine a relative increase of the diffusion coefficient.

Let us see now what are the consequences of equation (48) at large times and at short times respectively. At large times, since the dispersion $\sigma_{x}^{2}$ increases in time, the action of the quantum potential can be neglected and thus the solution of Eq. (48) tends asymptotically to the Einstein law $\sigma_{x}^{2}=2 D t$, which here reads as

$$
\sigma_{x}^{2}=\frac{\hbar n^{2} l_{p}^{2} \Delta \rho_{q v E_{0}}}{\mu} t .
$$

On the contrary, at small times the quantum term is dominant and one can neglect the unity in the brackets of Eq. (48). In this way, one obtains a solution in terms of the expression for the purely quantum diffusion

$$
\sigma_{x}^{2}=\hbar \sqrt{\frac{t}{m b}}
$$

which here reads

$$
\sigma_{x}^{2}=\hbar \sqrt{\frac{\hbar n^{2} l_{p}^{2} \Delta \rho_{q v E_{0}} t}{2 \mu m k_{B} T}} .
$$

At small times, in the thermodynamic limit, the background has the possibility to reach the maximum degree of non-locality expressed by the value 1 of the Bell length of the vacuum, where the constraint (31) holds. Therefore, the dispersion $\sigma_{x}^{2}$ in this condition becomes

$$
\left.\sigma_{x}^{2}=\hbar \sqrt{\hbar\left(\frac{16 \Delta \rho_{q v E_{0}}^{4} V^{2}}{\left[-\left\{\nabla \Delta \rho_{q v E_{0}}\right\}^{2}+\frac{1}{c^{2}}\left\{\frac{\partial}{\partial t} \Delta \rho_{q v E_{0}}\right\}^{2}-\Delta \rho_{q v E_{0}}\left\{\nabla^{2} \Delta \rho_{q v E_{0}} \frac{1}{c^{2}} \frac{\partial^{2}}{\partial t^{2}} \Delta \rho_{q v E_{0}}\right\}\right]}\right)}\right) \frac{l_{p}^{2} \Delta \rho_{q v E_{0}} t}{2 \mu m k_{B} T} .
$$

In the light of the formalisms (49), (51) and (52), a fundamental result of the approach to the Brownian motion developed in this chapter is the following. Both for large times (when the action of the quantum potential is negligible) and for small times (when the action of the quantum potential is dominant), the dispersion of the wave packet corresponding to the test particle is a collective property which emerges from the perturbative fluctuation of the quantum vacuum energy density and thus from the polarized vacuum characterized by a fluctuating viscosity. Therefore, the following rereading of the results regarding the dispersion of the wave packet in the Brownian motion of a test particle inside the $3 \mathrm{D}$ quantum vacuum model, becomes permissible. It is the specific interplay between the perturbative fluctuation of the quantum vacuum energy density -associated with the peculiar excited state of the 3D quantum vacuum interacting with the test particle- and the viscosity of the vacuum which implies that in some situations the dispersion of the wave packet is given by relation (49) and in some situations the dispersion of the wave packet is given by relation (51). In other words, in the model of the Brownian motion here presented, we can 
say that the dominance of quantum effects or the dominance of classical effects can be seen as collective behaviours which emerge from a more fundamental interplay between the perturbative fluctuation of the quantum vacuum energy density and the viscosity of the vacuum, having a specific behaviour; and, in the thermodynamic limit, at small times, in the condition of maximum degree of non-locality the dispersion assumes the peculiar form (52).

At the end of this section, we will make some considerations regarding numerical parameters which give rise to a Brownian motion at intergalactic level, in light of the results obtained at the end of Sec. 2. On the basis of the results obtained in [10], the total event rate of interaction of ordinary matter with the specific fluctuations of quantum vacuum energy density mimicking the action of dark matter may be expressed as follows:

$$
\begin{aligned}
R & =(Z+N)^{2} \rho_{q v e-N}^{D M} \frac{M_{T}}{M_{m o l}} \frac{\rho_{q v e-N}^{D M}}{M_{q v e-N}^{D M}} \bar{v}_{q v e-N}^{D M}=0.37 \\
& \times\left(\frac{Z+N}{12} \frac{\rho_{q v e-N}^{D M}}{10^{-31} \mathrm{~cm}^{2}} \frac{M_{T}}{10^{-3} \mathrm{~g}} \frac{\rho_{q v e-N}^{D M}}{0.3 \mathrm{GeV} / \mathrm{cm}^{3}}\right. \\
& \left.\times \frac{20 \mathrm{MeV}}{M_{q v e-N}^{D M}} \frac{\bar{v}_{q v e}^{D M}}{341 \mathrm{Km} / \mathrm{s}}\right) \frac{1}{s}
\end{aligned}
$$

where $Z$ and $N$ are the atomic number and the number of neutrons respectively, $\rho_{q v e-N}^{D M}$ is the average cross section of the virtual particles of the medium mimicking the action of dark matter with the target nucleon, $M_{T}$ is the target mass, $M_{m o l}$ is the molar mass of the target, $\rho_{q v e}^{D M}=0.3 \mathrm{GeV} / \mathrm{cm}^{3}$ is the assumed value for the energy density associated with the regions of quantum vacuum mimicking the action of dark matter, $\bar{v}_{q v E}^{D M}$ is the velocity of the region of quantum vacuum into consideration. Here, it must be emphasized that, although the interaction rate has units of inverse time, it does not correspond to a well-defined frequency since the hits occur at random intervals with random recoil momenta. As regards the interaction between the target mass and the virtual particles of the medium mimicking the action of dark matter, here two are the main sources of these events of interaction: neutrinos and hits from residual air molecules. The largest neutrino flux on Earth is from solar pp neutrinos with $\phi_{v} \approx 6 \cdot 10^{10} \mathrm{~cm}^{-2} \mathrm{~s}^{-1}$ at an energy of about $0.4 \mathrm{MeV}$ corresponding to a nucleon cross section to carbon of about $10^{-44}$ $\mathrm{cm}^{2}$. This means that, if one assumed a target material particle of $10^{-3} \mathrm{~g}$, in correspondence there is a neutrino event rate of $\mathrm{O}\left(10^{-14}\right) \mathrm{s}^{-1}$.

On the other hand, the differential event rate of air molecules hitting the target, after integrating over all velocities, is given by relation

$$
\begin{aligned}
\frac{d N}{d t} & =P A \sqrt{\frac{2}{\pi m k_{B} T}} \approx 8.3 \times 10^{9} \\
& \times\left(\frac{P}{10^{-10} \mathrm{mbar}} \sqrt{\frac{20 K}{T}} \frac{A}{\mathrm{~mm}^{2}}\right) \frac{1}{s}
\end{aligned}
$$

where $P$ is the pressure, $A$ is the surface of the target, $T$ is the temperature, $k_{B}$ is the Boltzmann constant. As regards the pressure provided by the air molecules, one can assume a vacuum system like in the LHC [36] or advanced LIGO [37] and for the temperature a cryonic system as in KAGRA [38]. By assuming a target surface of $1 \mathrm{~mm}^{2}$, the variance of the Gaussian distribution of the recoil momenta from virtual particles of the medium mimicking dark matter and the atmosphere, on the basis of [10], is given by the following relation

$$
\begin{aligned}
& \sigma_{q_{a t m}}^{2}=8 \sqrt{\frac{2}{\pi}} P A \delta t \sqrt{m k_{B} T} \approx 6.1^{-48} \\
& \times\left(\frac{P}{10^{-10} \mathrm{mbar}} \frac{A}{\mathrm{~mm}^{2}} \frac{\delta t}{0.1 \mathrm{~ns}} \sqrt{\frac{20 K}{T}}\right) \frac{\mathrm{Kg}^{2} \mathrm{~m}^{2}}{\mathrm{~s}^{2}}
\end{aligned}
$$

where $\delta t$ are the data bins, which are of $0.1 \mathrm{~ns}$ in common optical measurements. From data bin to data bin we hence expect a momentum uncertainty of order of $\sigma_{q_{a t m}} \approx$ $2.5 \times 10^{-24} \mathrm{Kg} \mathrm{m} / \mathrm{s}$. In every realistic experiment, we would expect such minimal resolution of the recoil momentum generated by the interaction between the residual gas and the virtual particles of the 3D quantum vacuum.

Moreover, one must take into account the uncertainty of the position measurement. The minimum position resolution is related with the minimum momentum resolution by relation

$$
q_{\text {min }}=M_{T} \omega_{0} d_{\min } .
$$

As regards the frequency $\omega_{0}$, since the rotating motion of the spiral galaxies reproducing the observed flattening of the orbital speeds is explained through a frequency of fluctuations of the viscosity of the polarized vacuum given by $\Omega_{n}=10^{-11} \mathrm{~s}^{-1}$, we can assimilate the frequency $\omega_{0}$ to this value. Therefore, by substituting $\omega_{0}=10^{-11} \mathrm{~Hz}$ in Eq. (56), in order to obtain a minimal momentum resolution of the order of $\mathrm{O}\left(10^{-23}\right) \mathrm{Kg} \mathrm{m} / \mathrm{s}$, we will have a minimum position resolution $d_{\min }=10^{-6} \mathrm{~m}$. This number corresponds to the uncertainty of position which gives rise to the maximum recoil momentum for quantum vacuum with a energy of a few $\mathrm{MeV}$ with an order one uncertainty from the velocity distribution so that we can expect to potentially see a signal for fluctuations of quantum vacuum energy density mimicking the action of dark matter masses above that. In other words, $d_{\min }=10^{-6} \mathrm{~m}$ can be considered the minimum resolution in the measurement of position which is associated with the Brownian motion of the polarized quantum vacuum reproducing the observed flattening of the orbital speeds of spiral 
galaxies. Furthermore, with this choice the background from the residual air molecules is very efficiently suppressed and we end up with an effective rate of interaction of ordinary matter with the specific fluctuations of quantum vacuum energy density mimicking the action of dark matter

$$
R_{\mathrm{atm}}^{\mathrm{cut}} \approx 5 \times 10^{-6} \mathrm{~Hz} .
$$

Taking account of the minimal position resolution $d_{\min }=$ $10^{-6} \mathrm{~m}$, the dispersion of the wave packet characterizing the Brownian motion of the test particle, which is connected directly to the perturbative fluctuation of the quantum vacuum energy density and thus to the polarized vacuum characterized by a fluctuating viscosity, given by relation (49) at large times, and by relation (51) at small times, respectively becomes

$$
\frac{\hbar n^{2} l_{p}^{2} \Delta \rho_{q v E_{0}}}{\mu} t=10^{-12} \mathrm{~m}^{2},
$$

at large times, and

$$
\hbar \sqrt{\frac{\hbar n^{2} l_{p}^{2} \Delta \rho_{q v E_{0}}}{2 \mu m k_{B} T}}=10^{-12} \mathrm{~m}^{2},
$$

at small times. We can say that Eqs. (58) and (59) can be considered the fundamental constraints which provide the conditions in order to have a Brownian motion at intergalactic level.

\section{Brownian motion in a three-dimensional quantum vacuum characterized by an ana- $\log$ FRW geometry}

The spatially-flat FRW spacetime, by predicting and describing cosmological phenomenon such as the Cosmic Microwave Background Radiation and formation of both large scale structure and light elements (hydrogen, deuterium, helium, etc.), can be considered the Standard Model of Cosmology. It provides the best scheme for the observational data to describe our large-scale homogeneous and isotropic expanding universe $[39,40]$. As a consequence, it is interesting to explore the quantum Brownian motion determined by quantum vacuum fluctuations in the context of a spatially-flat FRW spacetime.

In this regard, in the recent paper [41] Bessa and his collaborators Bezerra, Bezerra de Mello and Mota, by studying the effects of quantum scalar field vacuum fluctuations on scalar test particles in an analog model for the FRW spatially flat geometry, found that the particles can undergo Brownian motion with a nonzero mean squared velocity induced by the quantum vacuum fluctuations due to the time dependent background and the presence of the boundaries.

Here, by following the philosophy that underlies Bessa's research, we want to analyse the motion of a test particle in the context of our model of 3D quantum vacuum characterized by a FRW geometry and by a perturbative fluctuation of the quantum vacuum energy density. We will see how, in the picture of a spatially-flat FRW geometry, the motion of a scalar test particle in the 3D quantum vacuum undergoes Brownian motion determined by the quantum vacuum energy density fluctuations associated with the elementary RS processes of creation/annihilation of virtual particles.

By following the treatment of [41], we can say that the equation of motion in a FRW spatially-flat geometry, for a free particle, assumes the following form

$$
\frac{1}{a^{2}} \frac{d}{d t}\left(a^{2} u_{i}\right)=\frac{f_{i}}{m}
$$

while, if the particle is bounded, becomes

$$
\frac{d u_{i}}{d t}=\frac{f_{i}+f_{\mathrm{ext}}}{m}
$$

where $a$ is the scale factor of the FRW metric, $f_{i}$ is a quantum force linked with the fluctuations of the quantum vacuum energy density, $f_{\text {ext }}=2 m(\dot{a} / a) u^{i}$ is the external force responsible of the bound state of the particle. The fluctuating force in the FRW geometry may be expressed as

$$
f_{i}=q a^{-2} \partial_{i} \phi\left(\Delta \rho_{\text {perturbative }}\right),
$$

where $\phi\left(\Delta \rho_{\text {perturbative }}\right)$ is a scalar field depending on the perturbative fluctuation of the quantum vacuum energy density, $q$ is the mass of the particle. Equation (62) expresses the fact that, in the 3D quantum vacuum characterized by a FRW geometry, the force acting on the test particle, which is owed to the $3 \mathrm{D}$ quantum vacuum, is linked with the scale factor as well as the perturbative fluctuation of the quantum vacuum energy density.

Now, as regards the Brownian motion of a free particle in analog FRW geometry, by integrating (60) -and taking Eq. (62) into account- one obtains the following expression for the correlation function for the velocity in terms of the fluctuating force:

$$
\begin{aligned}
& \left\langle u_{i}\left(t_{1}, \Delta \rho_{\text {perturbative 1 }}\right) u_{i}\left(t_{2}, \Delta \rho_{\text {perturbative 2 }}\right)\right\rangle \\
& \quad=\frac{q^{2}}{m^{2} a^{4}} \iint d t_{1} d t_{2} \\
& \quad \times\left\langle\phi\left(t_{1}, \Delta \rho_{\text {perturbative 1 }}\right) \phi\left(t_{2}, \Delta \rho_{\text {perturbative 2 }}\right)\right\rangle_{\text {FRW }}
\end{aligned}
$$

Here, since the correlation function for the scalar field in the conformal 4-dimensional FRW spacetime is related to Minkowski flat spacetime by relation

$$
\begin{aligned}
& \left\langle\phi\left(\eta_{1}, \Delta \rho_{\text {perturbative 1 }}\right) \phi\left(\eta_{2}, \Delta \rho_{\text {perturbative 2 }}\right)\right\rangle_{\mathrm{FRW}} \\
& \quad=a^{-1}\left(\eta_{1}\right) a^{-1}\left(\eta_{2}\right) \\
& \quad \times\left\langle\phi\left(\eta_{1}, \Delta \rho_{\text {perturbative 1 }}\right) \phi\left(\eta_{2}, \Delta \rho_{\text {perturbative 2 }}\right)\right\rangle_{\text {flat }},
\end{aligned}
$$

where $\eta$ is the conformal time, defined as $d t=a(\eta) d \eta$, the subscript in the right-hand side denotes that the vacuum fluctuation is taken in a flat spacetime, Eq. (63) becomes 


$$
\begin{aligned}
& \left\langleu _ { i } ( t _ { 1 } , \Delta \rho _ { \text { perturbative 1 } } ) u _ { i } \left( t_{2}, \Delta \rho_{\text {perturbative 2 })\rangle}\right.\right. \\
& \quad=\frac{q^{2}}{m^{2} a^{4}} \partial i_{1} \partial i_{2} \iint d \eta_{1} d \eta_{2} \\
& \quad \times\left\langle\phi\left(\eta_{1}, \Delta \rho_{\text {perturbative } 1}\right) \phi\left(\eta_{2}, \Delta \rho_{\text {perturbative 2 }}\right)\right\rangle_{\text {flat }}
\end{aligned}
$$

Taking account that $v=a u$, Eq. (65), in terms of $v$, may be written in the form

$$
\begin{aligned}
& \left\langle v_{i}\left(t_{1}, r_{1}\right) v_{i}\left(t_{2}, r_{2}\right)\right\rangle=\frac{q^{2}}{m^{2} a^{2}} \partial i_{1} \partial i_{2} \iint d \eta_{1} d \eta_{2} \\
& \quad \times\left\langle\phi\left(\eta_{1}, \Delta \rho_{\text {perturbative } 1)}\right) \phi\left(\eta_{2}, \Delta \rho_{\text {perturbative } 2)}\right)\right\rangle_{\text {flat }}
\end{aligned}
$$

Equations (62-66), in light of the mathematical formalism developed in Sec. 3, imply that, even if a particle is free, the presence of the perturbative fluctuation of the quantum vacuum energy density -contained inside the scalar field $\phi-$ generate a Brownian motion for these particles.

As for the Brownian motion for bounded particles in the spatially-flat FRW geometry, by integrating Eq. (61) with a null initial velocity, we obtain

$$
u^{i}\left(t_{f}\right)=\frac{1}{m} \int f^{i}\left(t, \Delta \rho_{\text {perturbative }}\right),
$$

where $t_{f}$ is the final time and the correlation function for the coordinate velocity is

$$
\begin{aligned}
& \left\langle u_{i}\left(t_{f}, \Delta \rho_{\text {perturbative 1 }}\right) u_{i}\left(t_{f}, \Delta \rho_{\text {perturbative } 2)}\right)\right. \\
& =\frac{1}{m^{2}} \iint d t_{1} d t_{2} \\
& \times\left\langle f^{i}\left(t_{1}, \Delta \rho_{\text {perturbative } 1}\right) f^{i}\left(t_{2}, \Delta \rho_{\text {perturbative } 2)}\right)\right\rangle_{\text {FRW }}
\end{aligned}
$$

By considering a fluctuating force (62) which satisfies the constraints $\left\langle f^{i}\left(t_{1}, \Delta \rho_{\text {perturbative 1 }}\right) f^{i}\left(t_{2}, \Delta \rho_{\text {perturbative 2 }}\right)\right\rangle_{\text {FRW }} \neq$ 0 and $\left\langle f^{i}\left(t, \Delta \rho_{\text {perturbative }}\right)\right\rangle_{\mathrm{FRW}}=0$, from Eq. (68) one gets

$$
\begin{aligned}
& \left\langle u_{i}\left(t_{1}, \Delta \rho_{\text {perturbative 1 }}\right) u_{i}\left(t_{2}, \Delta \rho_{\text {perturbative } 2)}\right)\right. \\
& =\frac{q^{2}}{m^{2}} \partial_{i 1} \partial_{i 2} \iint d t_{1} d t_{2} a^{-1}\left(t_{1}\right) a^{-1}\left(t_{2}\right) \\
& \quad \times\left\langle\phi\left(\eta_{1}, \Delta \rho_{\text {perturbative } 1}\right) \phi\left(\eta_{2}, \Delta \rho_{\text {perturbative } 2)}\right) .\right.
\end{aligned}
$$

When Eq. (66) is expressed in terms of the conformal time, $d t=a(\eta) d \eta$, and in terms of the massless two point function given by relation (64), it provides the following expression for the velocity dispersion of the bounded particles

$$
\begin{aligned}
& \left\langle u_{i}\left(\eta_{1}, \Delta \rho_{\text {perturbative 1 }}\right) u_{i}\left(\eta_{2}, \Delta \rho_{\text {perturbative } 2)}\right)\right. \\
& \quad=\frac{q^{2}}{m^{2}} \partial_{i 1} \partial_{i 2} \iint d \eta_{1} d \eta_{2} a^{-2}\left(\eta_{1}\right) a^{-2}\left(\eta_{2}\right) \\
& \quad \times\left\langle\phi\left(\eta_{1}, \Delta \rho_{\text {perturbative } 1}\right) \phi\left(\eta_{2}, \Delta \rho_{\text {perturbative } 2)}\right) .\right.
\end{aligned}
$$

In this case, the proper velocity is given by

$$
\begin{aligned}
& \left\langle v_{i}\left(\eta_{1}, \Delta \rho_{\text {perturbative 1 }}\right) v_{i}\left(\eta_{2}, \Delta \rho_{\text {perturbative } 2)}\right)\right. \\
& =\frac{q^{2} a^{2}}{m^{2}} \partial_{i 1} \partial_{i 2} \iint d \eta_{1} d \eta_{2} a^{-2}\left(\eta_{1}\right) a^{-2}\left(\eta_{2}\right) \\
& \quad \times\left\langle\phi\left(\eta_{1}, \Delta \rho_{\text {perturbative } 1}\right) \phi\left(\eta_{2}, \Delta \rho_{\text {perturbative } 2)}\right) .\right.
\end{aligned}
$$

As regards the proper velocity, by comparing Eqs. (66) and (71), we can note that, differently from free particles, in the case of bounded particles, the scale factor appears in the integrand of Eq. (71). This is a consequence of the fact that, for bounded particles, there is also an external force responsible of the bounded state of the particle. This external force cancels out locally the effects of the expansion and it is just this cancellation that makes the time dependent scale factor appear in the integrand of Eq. (71), which represents the quantum dispersion in the velocity for bound particles, and not appear in Eq. (66), which represents the quantum dispersion in the velocity for the free particles.

Moreover, we must observe that the scalar field depending on the perturbative fluctuation of the quantum vacuum energy density $\phi\left(\Delta \rho_{\text {perturbative }}\right)$ satisfies the Klein-Gordon equation

$$
\begin{aligned}
\left(\frac{1}{\sqrt{|\sqrt{g}|}}\right) \partial_{\mu}\left[\sqrt{|\sqrt{g}|} g^{\mu \nu} \partial_{\nu}\right. \\
+\xi R] \phi\left(\Delta \rho_{\text {perturbative }}\right)=0
\end{aligned}
$$

where $g^{\mu \nu}$ is the metric characterizing the FRW geometry, $g=\operatorname{det}\left(g^{\mu \nu}\right), R$ is the Ricci scalar, $\xi$ is the curvature coupling. The solution $\varphi\left(\Delta \rho_{\text {perturbative }}\right)$ of Eq. (62) may be expressed as

$$
\begin{aligned}
& \phi\left(\Delta \rho_{\text {perturbative }}\right)=\left(\frac{\hbar p \eta n^{2} l_{p}^{2} \Delta \rho_{q v E_{0}}}{16 \pi^{2} \mu c^{2}}\right)^{1 / 2} \\
& \quad \times \exp \left(-i \frac{2 \mu c^{2}}{\hbar n^{2} l_{p}^{2} \Delta \rho_{q v E_{0}}} t\right. \\
& \left.+\frac{1}{c \hbar}\left[i \frac{2 \mu c^{2}}{n^{2} l_{p}^{2} \Delta \rho_{q v E_{0}}} x-\frac{2 \mu c^{2}}{n^{2} l_{p}^{2} \Delta \rho_{q v E_{0}}}\left|x-x_{0}\right|\right]\right),
\end{aligned}
$$

where $p$ is a parameter depending on the viscosity of the vacuum and the Newton gravitational constant on the basis of relation $p=\left(1-4 G 2 \mu c^{2} / n l_{p}^{3} \Delta \rho_{q v E_{0}}\right)^{-1}, x_{0}$ is the maximum of the amplitude of the wave function. In summary, in the light of the Eqs. (63)-(73), we can conclude that a test particle with mass $m$ moving with proper velocity $v$ in a FRW geometry exhibits a Brownian motion characterized by oscillations in the physical vacuum as a consequence of the action of the perturbative fluctuation of the quantum vacuum energy density which is contained in the scalar field $\phi\left(\Delta \rho_{\text {perturbative }}\right)$. 


\section{Conclusions and perspectives}

Brownian motion emerges as a collective property originated by a fundamental 3D quantum vacuum characterized by a variable energy density as a consequence of the interaction between a test particle and an opportune perturbative fluctuation of the quantum vacuum energy density associated with a fluctuating viscosity. The quantum diffusion associated with the Brownian motion of a test particle is guided by the quantum potential and is influenced by the mediatory action of the perturbative fluctuation of the quantum vacuum energy density. The perturbative fluctuation of the quantum vacuum energy density provides a correction in terms of dissipative, dispersive effects into the Brownian motion of the particle. As a consequence, the dispersion turns out to be a collective property generated by the ultimate properties of the polarized 3D quantum vacuum. The interplay between the perturbative fluctuation of the quantum vacuum energy density -associated with the peculiar excited state of the 3D quantum vacuum interacting with a test particle- and the viscosity of the vacuum, having a specific behaviour, can be considered the ultimate visiting card which generates Brownian motion at intergalactic level, reproducing the observed flattening of the orbital speeds of spiral galaxies. Moreover, the correlation function for the velocity of a Brownian particle in a FRW spatially-flat geometry, turns out to depend on a scalar field which is directly correlated with the perturbative fluctuation of the quantum vacuum energy density.

An interesting perspective introduced by our model lies in the interpretation of the deviation of the dynamics of galaxies from the Newtonian behaviour on large scales in terms of the Brownian motion generated by a perturbative fluctuation of the energy density of the ultimate 3D quantum vacuum characterized by processes of creation/annihilation of virtual particles. In fact, another relevant topic to be addressed inside our model would be the treatment of the anomalous rotations of other type of galaxies. In order to extend our model to other type of galaxies, according to the author, a possible way could be represented by some fruitful considerations made recently by Jurisch [42] that the phenomenon of anomalous rotation can be described by the equilibrium state of a stochastic process in a picture where the weak-interacting limit of a metric-skew-tensor-gravity theory can be derived from the ordinary Brownian motion with spherical symmetry. In line with the treatment of Jurisch, one can start from a spherical model of the quantum vacuum energy density of the form

$$
\Delta \rho_{q v E_{0}}(r)=\Delta \rho_{q v E_{0}}\left(\frac{r}{R_{c}+r}\right)^{3 \beta}
$$

where $R_{c}$ denotes the radius of the core and $\beta$ describes the different types of the phenomenology associated with the different types of the galaxies. In particular, $\beta=1$ corresponds to high-surface-brightness galaxies, while $\beta=2$ corresponds to low-surface-brightness galaxies and dwarf galaxies. The rotational curve can be computed by equating the gravitational acceleration with the centripetal acceleration

$$
\frac{v^{2}}{r}=\frac{V}{c^{2}} \Delta \rho_{q v E_{0}}\left(\frac{r}{R_{c}+r}\right)^{3 \beta} \frac{\partial \phi}{\partial r},
$$

which gives

$$
v(r)=\sqrt{r \frac{V}{c^{2}} \Delta \rho_{q v E_{0}}\left(\frac{r}{R_{c}+r}\right)^{3 \beta} \frac{\partial \phi}{\partial r}},
$$

where $\phi$ is the gravitational potential. By following [42], the form of the gravitational potential $\phi$ may be obtained by starting from the following equation describing the Brownian correction to the classical trajectory of the galaxy:

$$
d r_{B}(t)=-\gamma d t+\sqrt{D} d W(t)
$$

where $d r_{B}(t)$ is the Brownian correction of the classical trajectory, $d W(t)$ is a Gaussian Wiener-process, $D$ is the diffusion coefficient given by Eq. (11) and $\gamma$ is a drift-parameter (having the dimension of velocity) that determines skewness in the sense that a test particle tends to move in the direction of the drift. After some mathematical manipulations, the gravitational potential which allows us to reproduce the anomalous rotation of galaxies turns out to be given by relation

$$
\begin{aligned}
\varphi(r) & =-G \frac{V}{c^{2} r} \Delta \rho_{q v E_{0}}+G \sqrt{\frac{V}{c^{2}} \Delta \rho_{q v E_{0}} \frac{\mu \hbar}{n^{2} l_{p}^{2} \Delta \rho_{q v E_{0}}}} \\
& \times \frac{\exp \left(-r / r_{0}\right)}{r} .
\end{aligned}
$$

A model based on this gravitational potential (78) can reproduce the flatness of the tail of the velocity $v(r)$ over a large distance, and the heavy attraction it provides, which corresponds to the drift-motion of the Brownian trajectory towards the centre of gravity, has the effect to generate a flat rotation curve also on large distance. As regards the cosmological perspectives opened by this type of approach, further research will give you more information.
1. R. Brown, A brief Account of Microscopical Observations made in the Months of June, July, and August, 1827, on the Particles contained in the Pollen of Plants; and on the general Existence of active Molecules in Organic and Inorganic Bod- ies, Philos. Mag. 4 (1828) 161, https://doi.org/10. 1080/14786442808674769; Ann. Phys. 90 (1828) 294, https://doi.org/10.1002/andp.18280901016 
2. A. Einstein, Über die von der molekularkinetischen Theorie der Wärme geforderte Bewegung von in ruhenden Flüssigkeiten suspendierten Teilchen, Ann. Phys. 322 (1905) 549, https : //doi.org/10.1002/andp.19053220806.

3. W. Sutherland, LXXV. A dynamical theory of diffusion for non-electrolytes and the molecular mass of albumin, Philos. Mag. 9 (1905) 781, https://doi.org/10.1080/ 14786440509463331

4. M. von Smoluchowski, Zur kinetischen Theorie der Brownschen Molekularbewegung und der Suspensionen, Ann. Phys. 326 (1906) 756, https://doi.org/10.1002/andp. 19063261405

5. P. Langevin, On the theory of Brownian motion, C. R. Acad. Sci. 146 (1908) 530.

6. J. Perrin, L'agitation moléculaire et le mouvement brownien, $C$. R. Acad. Sci. 146 (1908) 967.

7. J. Perrin, Mouvement Brownien et Réalité Moléculaire, Ann. Chim. Phys. 18 (1909) 5.

8. I. Nordlund, A New Determination of Avogadro's Number from Brownian Motion of Small Mercury Spherules, Z. Phys. Chem. 87 (1914) 40.

9. E. Kappler, Versuche zur Messung der AvogadroLoschmidtschen Zahl aus der Brownschen Bewegung einer Drehwaage, Ann. Phys. 11 (1931) 233.

10. T. Chen, R. Primulando, and M. Spinrath, Dark matter induced Brownian motion, Eur. Phys. J. C 80 (2020) 519, https: //doi.org/10.1140/epjc/s10052-020-8066-8

11. D. Fiscaletti and A. Sorli, Perspectives about quantum mechanics in a model of a three-dimensional quantum vacuum where time is a mathematical dimension, SOP Trans. Theor. Phys. 1 (2014) 11

12. D. Fiscaletti and A. Sorli, Space-time curvature of general relativity and energy density of a three-dimensional quantum vacuum, Ann. Univ. Mariae Curie-Sklodowska Sectio AAA 69 (2014) 53, https://doi.org/10.17951/aaa.2014. 69.55

13. D. Fiscaletti, The Timeless Approach, 1st ed. (World Scientific, Singapore, 2016).

14. D. Fiscaletti, About Dark Energy and Dark Matter in a Three-Dimensional Quantum Vacuum Model, Found. Phys. 46 (2016) 1307, https://doi.org/10.1007/ s10701-016-0021-z

15. D. Fiscaletti and A. Sorli, About a Three-Dimensional Quantum Vacuum as the Ultimate Origin of Gravity, Electromagnetic Field, Dark Energy and Quantum Behavior, Ukr. J. Phys. 61 (2019) 413, https://doi.org/10.15407/ ujpe61.05.0413

16. D. Fiscaletti, Dynamic Quantum Vacuum and Relativity, Ann. Univ. Mariae Curie-Sklodowska Sectio AAA 71 (2016) 11, https://doi.org/10.17951/aaa.2016.71.11

17. D. Fiscaletti, What is the Actual Behaviour of the Electron? From Bohm's Approach to the Transactional Interpretation to A Three-Dimensional Timeless Non-Local Quantum Vacuum, Electron. J. Theor. Phys. 35 (2016) 13.
18. D. Fiscaletti and A. Sorli, About Electroweak Symmetry Breaking, Electroweak Vacuum and Dark Matter in a New Suggested Proposal of Completion of the Standard Model in Terms Of Energy Fluctuations of a Timeless Three- Dimensional Quantum Vacuum, Quantum Phys. Lett. 5 (2016) 33, https: // doi. org/10.18576/qpl/050302

19. D. Fiscaletti and A. Sorli, Quantum vacuum energy density and unifying perspectives between gravity and quantum behaviour of matter, Ann. Fond. Louis de Broglie 42 (2017) 251.

20. D. Fiscaletti and A. Sorli, Quantum Relativity: Variable Energy Density of Quantum Vacuum as the Origin of Mass, Gravity and the Quantum Behaviour, Ukr. J. Phys. 63 (2018) 623, https://doi.org/10.15407/ujpe63.7.623.

21. I. Licata and L. Chiatti, Timeless Approach to Quantum Jumps, Quanta 4 (2015) 10, https://doi.org/10.12743/ quanta.v4i1.31

22. L. Chiatti and I. Licata, Particle model from quantum foundations, Quantum Stud.: Math. Found. 4 (2017) 181, https: //doi.org/10.1007/s40509-016-0094-6

23. D. Fiscaletti, About dark matter as an emerging entity from elementary energy density fluctuations of a three-dimensional quantum vacuum, J. Theor. Appl. Phys. 14 (2020) 203, https://doi.org/10.1007/ s40094-020-00379-0

24. V. I. Sbitnev, Dark matter is a manifestation of the vacuum Bose-Einstein condensate, arXiv:1601.04536.

25. M. Milgrom, A modification of the Newtonian dynamics as a possible alternative to the hidden mass hypothesis, Astrophys. J. 270 (1983) 365.

26. M. Milgrom, A modification of the Newtonian dynamics: Implications for galaxies, Astrophys. J. 270 (1983) 371.

27. M. Milgrom, MOND laws of galactic dynamics, Mon. Not. R. Astron. Soc. 437 (2014) 2531, https://doi.org/10. $1093 / \mathrm{mnras} / \mathrm{stt} 2066$

28. A. R. Penner, Induced energy polarization of the vacuum as the source of the baryonic Tully-Fisher relationship and the Pioneer anomaly, Can. J. Phys. 90 (2012) 315, https: / /doi.org/ $10.1139 / \mathrm{p} 2012-029$.

29. A. R. Penner, Induced energy polarization of the vacuum and the rotational curve for the Galaxy, Can. J. Phys. 91 (2013) 126, https://doi.org/10.1139/cjp-2012-0300

30. A. R. Penner, Induced energy polarization of the vacuum and the rotational curves of spiral galaxies, Can. J. Phys. 91 (2013) 610, https://doi.org/10.1139/cjp-2013-0060

31. A. R. Penner, Induced energy polarization of the vacuum and the Coma cluster, Can. J. Phys. 91 (2013) 1114, https: //doi.org/10.1139/cjp-2013-0294

32. A. R. Penner, Induced energy polarization of the vacuum and the resulting cosmology, arXiv: 1405.5165 .

33. M. Roshan and S. Rahvar, Evolution of spiral galaxies in nonlocal gravity, Astrophys. J. 872 (2019) 6, https : / doi.org/ $10.3847 / 1538-4357 / a a f c 2 a$.

34. R. Tsekov, Nonlinear Theory of Quantum Brownian Motion, Int. J. Theor. Phys. 48 (2009) 85, https://doi.org/10. 1007/s10773-008-9784-0 
35. G. Grössing, The vacuum fluctuation theorem: Exact Schrödinger equation via nonequilibrium thermodynamics, Phys. Lett. A 372 (2008) 4556, https://doi.org/10. $1016 / j \cdot$ physleta.2008.05.007

36. LHC Guide, https://cds.cern.ch/record/ 2255762

37. J. Aasi et al. (LIGO Scientific Collaboration), Advanced LIGO, Class. Quantum Grav. 32 (2015) 074001, https://doi. org/10.1088/0264-9381/32/7/074001

38. K. Somiya, Detector conficuration of KAGRA the Japanese cryogenic gravitational-wave detector, Class. Quantum Grav. 29 (2012) 124007, https: //doi.org/10.1088/0264-9381/29/12/124007
39. P. A. R. Ade et al. (Planck Collaboration), Planck 2015 results: XVIII. Background geometry and topology of the Universe, Astron. Astrophys. 594 (2016) A18, https://doi.org/10. 1051/0004-6361/201525829

40. S. Weinberg, Cosmology (Oxford University Press, Oxford, 2008).

41. C. H. G. Bessa, V. B. Bezerra, E. R. Bezerra de Mello, and H. F. Mota, Quantum Brownian motion in an analog Friedmann-Robertson-Walker geometry, Phys. Rev. D 95 (2017) 085020, https://doi.org/10.1103/ PhysRevD.95.085020

42. A. Jurisch, Brownian motion approach to anomalous rotation of galaxies, arXiv:1912.00737. 